\title{
Long non-coding RNA HR1 participates in the expression of SREBP-1c through phosphorylation of the PDK1/AKT/FoxO1 pathway
}

\author{
DUAN LI ${ }^{1,2^{*}}$, LIWEI GUO $^{3}$, BAOGUO DENG $^{1}$, MIN LI $^{1}$, TINGTING YANG $^{1}$, FAN YANG $^{1}$ and ZHIJUN YANG ${ }^{4 *}$ \\ ${ }^{1}$ Department of Microbiology, School of Basic Medical Sciences, Xinxiang Medical University, Xinxiang, Henan 453003; \\ ${ }^{2}$ Department of Psychiatry, The Second Affiliated Hospital of Xinxiang Medical University, Xinxiang, Henan 453002; \\ ${ }^{3}$ School of Forensic Medicine; ${ }^{4}$ Department of Chemistry, School of Basic Medical Sciences, \\ Xinxiang Medical University, Xinxiang, Henan 453003, P.R. China
}

Received February 6, 2018; Accepted June 20, 2018

DOI: $10.3892 / \mathrm{mmr} .2018 .9278$

\begin{abstract}
Sterol regulatory element binding protein-1c (SREBP-1c), which serves an essential role in the process of fat synthesis, is a key adjustment factor that regulates the dynamic balance of lipid metabolism. SREBP-1c activates the transcription of multiple genes encoding for enzymes involved in the synthesis of triglycerides (TG) and fatty acids (FA) and accelerates lipid synthesis. Previous analysis indicated that long non-coding RNA HCV regulated 1 (lncHR1) participates in lipid metabolism in vivo and regulates the level of SREBP-1c protein. However, the mechanism of 1 ch HR 1 in regulating SREBP-1c levels has not been revealed. In the present study, a fatty degeneration cell model was used to study how 1ncHR1 regulates the SREBP-1c protein at the cellular level. Furthermore TG accumulation was assessed according to morphological analysis. Reverse transcription-quantitative polymerase chain reaction and western blotting were used to detected the expression of SREBP-1c. An activator and an inhibitor of phosphoinositide 3-kinase/AKT phosphorylation (IGF-1 and LY294002, respectively) were used to study the
\end{abstract}

Correspondence to: Dr Duan Li, Department of Microbiology, School of Basic Medical Sciences, Xinxiang Medical University, 601 Jinsui Road, Xinxiang, Henan 453003, P.R. China

E-mail: liduan1979@163.com

*Contributed equally

Abbreviations: 1ncRNA, long non-coding RNA; SREBP-1c, sterol regulatory element binding protein-1c; OA, oleic acids; LXR $\alpha$, liver $\mathrm{X}$ receptor $\alpha$; SCAP, SREBP cleavage-activating protein; DAPI, 4',6-diamidino-2-phenylindole; FA, fatty acid; FAS, fatty acid synthase; TG, triglyceride; ACC $\alpha$, acetyl-CoA carboxylase $\alpha$; RT-qPCR, reverse transcription-quantitative polymerase chain reaction

Key words: lncHR1, SREBP-1c, PDK1/AKT/FoxO1, triglyceride, lipid metabolism effect of lncHR1 on this pathway. It was verified that lncHR1 regulated SREBP-1c levels and the phosphorylation of AKT in the steatosis cell model. Detailed molecular mechanisms mediated by lncHR1 were associated with the phosphorylation AKT/FoxO1 in Huh7 cell lines. Simultaneously, lncHR1 affected the location of FoxO1 inside and outside of the nucleus. Furthermore, the phosphorylation of PDK1 upstream of AKT was regulated through overexpression or knockdown lncHR1, as determined by western blotting. Taken together, these data show that lncHR1 inhibits SREBP-1c levels through the phosphorylation of the PDK1/AKT/FoxO1 axis.

\section{Introduction}

Sterol regulatory element binding protein-1c (SREBP-1c) is one of SREBP family member and regulate the expression of lipogenic genes $(1,2)$. Recent in vivo studies suggest that SREBP-1c is central to most hepatic lipogenic genes. SREBP-1c is involved in encoding enzymes that catalyze various steps in the fatty acid (FA) and triglyceride (TG) synthesis pathway, including fatty acid synthase (FAS) (3) and acetyl-CoA carboxylase $\alpha$ (ACC $\alpha$ ) (4). SREBP-1c facilitates FAS synthesis and incorporates FA into TG (5). Abnormally high SREBP-1c levels may cause hepatic TG accumulation and potentially induce other lipid disorders (6).

SREBP-1c is also a target of insulin, which activates transcription of the gene encoding SREBP1 by partially increasing the activity of liver X receptor $\alpha(\mathrm{LXR} \alpha)$ (7). SREBP-1c promoter activity is partially upregulated through $\mathrm{LXR} \alpha / \mathrm{RXR}$ heterodimer binding to the promoter area of LXR elements (LXREs) (8). However, a study by Kamei's revealed that a LXR $\alpha /$ RXR heterodimer binding to LXREs promotes was antagonized by forkhead Box O1 (FoxO1) (9). FoxO1, which belongs to the a FoxO transcription factor family, is typically regarded as a tumor suppressor and lies downstream of the phosphoinositide 3-kinase (PI3K)/AKT signaling pathway. A study by Deng et al (10) indicated that FoxO1 was associated with the SREBP-1c promoter and negatively regulated srebp1 gene expression via multiple mechanisms including modification of the promoter binding sites of Sp1 and SREBP-1c itself. 
In addition, the present study supported the hypothesis that increased FoxO1 levels decreased the level of SREBP-1c.

Long noncoding RNA (lncRNA) are transcribed RNA molecules that lack an open reading frame and are longer than 200 nucleotides (11). LncRNA regulate gene expression by diverse mechanisms (12). Recent evidence also suggests that lncRNA are abnormally expressed in liver cancer (13); an example is highly upregulated in liver cancer (HULC) (14).

Long non-coding RNA lncHR1 (HCV regulated 1, termed lncHR1), was first reported as upregulated in Huh7 cell infected by hepatitis $\mathrm{C}$ virus (HCV). As a new long non-coding RNA, IncHR1 exhibited obviously regulatory functions via SREBP-1c, the accumulation of TG and lipid droplets in cells, and in transgenic mouse model (15). Metastasis-associated lung adenocarcinoma transcript 1 (MALAT1) has been reported to regulate SREBP-1c expression at a post-transcriptional level had been verified (16). However, the molecular mechanisms behind the regulating of IncHR1 by SREBP-1c have yet to be determined.

In this study, we initially studied the molecular mechanisms behind the regulation of SREBP-1c by lncHR1. The results showed that lncHR1 may affect the phosphorylation of the PDK1/AKT/FoxO1 signaling pathway, subsequently regulating SREBP-1c protein levels in hepatocellular carcinoma lines. Thus, our study offered a new information regarding IncRNA regulation of SREBP-1c through the AKT/FoxO1 signaling pathway and provided a practical and efficient platform for studying the function of lncRNA in lipid metabolism.

\section{Materials and methods}

Cells and reagents. The Huh7 human hepatoma cell line was purchased from Apath, Inc. (Brooklyn, NY, USA) (17). The cells were cultured in DMEM medium from Thermo Fisher Scientific, Inc. (Waltham, MA, USA), supplemented with $10 \%$ FBS, $1 \%$ NEAA, $1 \%$ penicillin, and $1 \%$ streptomycin at $37^{\circ} \mathrm{C}$ and $5 \% \mathrm{CO}_{2}$. Antibodies for phospho-AKT (no. 9275), FoxO1 (no. 2280) and p-FoxO1 (no. 84192) were obtained from Cell Signaling Technology, Inc. (Danvers, MA, USA), antibodies for SREBP-1c (sc-13551) were from Santa Cruz Biotechnology, Inc., (Dallas, TX, USA), $\beta$-actin (A3854) was from Sigma-Aldrich (Merck KGaA, Darmstadt, Germany), and the Histone 3 (AF0009), IGF-1 (P5502) and LY294002 (S17375502) were from Beyotime Institute of Biotechnology (Shanghai, China). Horseradish peroxidase (HRP)-conjugated secondary antibodies (323-001-021) were from Jackson Immuno-Research (West Grove, PA, USA).

Cell culture, plasmids and transient transfection. LncHR1 was cloned into the $B g l \mathrm{II}$ and XhoI sites of a pMIR-Empty-DFT overexpression vector. shRNA targeting lncHR1 were cloned into the $B g l \mathrm{II}$ and $\mathrm{XhoI}$ sites of the pQSUP-R plasmid vector. The efficiency of overexpression or knockdown plasmids has been verified as previously described (15).

Cultured cells were inoculated in 24 well plates with $10 \%$ FBS medium and incubated overnight at $37^{\circ} \mathrm{C}$ and $5 \% \mathrm{CO}_{2}$ until the cells grew to $80 \%$ confluence. Before transfection, cell medium was replaced with fresh medium not containing with FBS for $1 \mathrm{~h}$. DNA $(1 \mu \mathrm{g})$ plasmids were diluted with serum-free DMEM medium and $2 \mu 1$ of the transfection reagent D293, which was diluted with serum-free DMEM medium, and then mixed well. The diluted transfection reagents were added to the diluted plasmid by drop by drop, gently mixed and then kept at room temperature for $15 \mathrm{~min}$. The suspension of the plasmid and the transfection reagent was added into the transfected cells evenly with gently agitation to ensure even distribution. The medium was replaced with fresh medium containing 10\% FBS after 5-8 h of transfection. After $48 \mathrm{~h}$ of continuous culture, cells were collected and then used for experiments.

\section{Oil red $O$ staining}

Oil red $O$ staining was done using the following steps. Huh7 cells were inoculated in the 24 well plates with the sterilized round glass slices and then carried out routine cell processing. Pretreated cells were fixed with 3\% paraformaldehyde for $10 \mathrm{~min}$ at room temperature and then were permeabilized with $0.4 \%$ Triton X-100 for $10 \mathrm{~min}$ at room temperature. Pretreated cells were stained for $1 \mathrm{~h}$ with freshly diluted $0.5 \mathrm{mM}$ oil red $\mathrm{O}$ dissolved in $60 \%$ isopropanol. Stained cells were washed with $50 \%$ ethyl alcohol twice for $5 \mathrm{~min}$ and then was washed with PBS twice for $5 \mathrm{~min}$. When washing the second times, PBS with 4',6-diamidino-2-phenylindole (DAPI) was added to stain the nuclei. Last, at least 5 random fields were observed under a Leica TCS-SL confocal microscope. The intensity was quantified with Image J software (NIH).

Western blotting and immunofluorescence analysis. Treated cells were collected and washed twice with PBS. For cell lysate preparation, the monolayer of plates cells was lysed with lysis buffer (50 mM Tris- $\mathrm{HCl}, \mathrm{pH} 7.5,150 \mathrm{mM} \mathrm{NaCl}$, $0.5 \%$ Nonidet P-40, $50 \mathrm{mM} \mathrm{NaF}, 1 \mathrm{mM} \mathrm{Na}_{3} \mathrm{VO}_{4}, 5 \mathrm{mM}$ $\beta$-glycerophosphate, $1 \mathrm{mM}$ dithiothreitol, and $1 \mathrm{mM}$ phenylmethylsulfonyl fluoride). The lysate was clarified by centrifuging at $14,000 \mathrm{x}$ g for $20 \mathrm{~min}$. Samples were mixed with $2 \mathrm{X}$ SDS loading buffer, boiled, and loaded onto a $10-12 \%$ polyacrylamide gel. After electrophoresis, the proteins were transferred onto a polyvinylidene difluoride membrane (Pall Corporation, East Hills, NY, USA). The resulting blots were blocked with $5 \%$ bovine serum albumin (BSA) for phosphoprotein antibodies, or $10 \%$ skim milk for non-phosphoprotein antibodies for $1 \mathrm{~h}$ and incubated with the selected primary antibody overnight at $4^{\circ} \mathrm{C}$. The secondary antibody used in the immunoblot was a 1:2,000 or 1:5,000 dilution of HRP-linked anti-IgG. ECL reagent (Amersham Biosciences, England, UK) was used as the substrate for detection, and the membrane was exposed to an x-ray film for visualization. The density of the bands was quantified using the NIH image software and were normalized to the density of the control band.

The nuclear-cytosol fractionation experiments were performed using a Nuclear-Cytosol Extraction kit (Applygen Technologies, Inc., Beijing, China). Western blotting was performed to quantify protein according to standard protocols published in the literature. Cells were mounted on glass slides and the immunofluorescence assay was performed as previously described (2). Images were captured with a Leica TCS-SL confocal microscope.

TG quantification. Intracellular TG were measured with a TG Assay kit from Applygen Technologies, Inc., according to the 
A

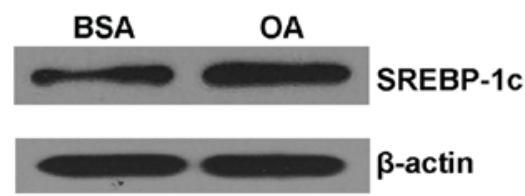

B

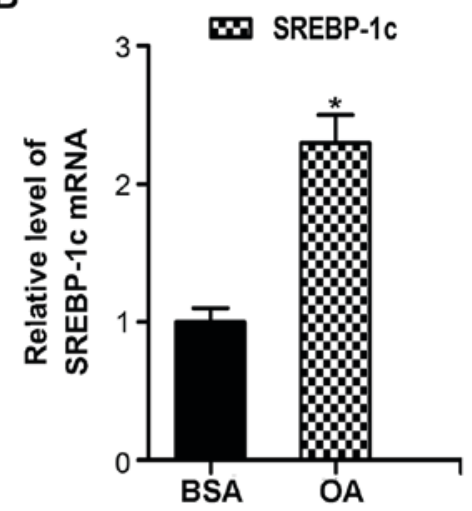

C

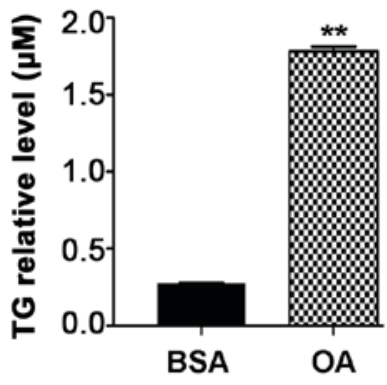

D

Huh7+BSA Huh7+OA

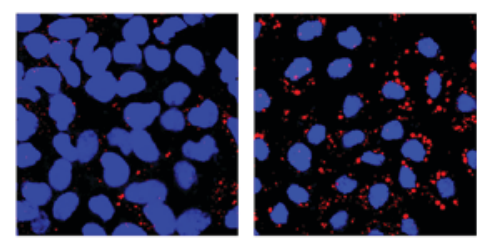

E

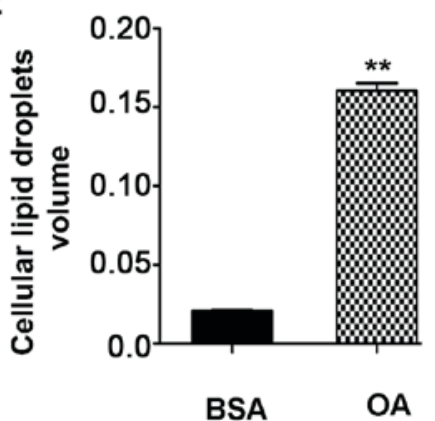

Figure 1. The model of OA induces hepatic cell triglyceride accumulation in Huh7 cells. (A and B) In Huh7 cells, OA treatment for $24 \mathrm{~h}$ significantly increased the level of SREBP-1c protein and the expression of SREBP-1c mRNA compared with control cells. (C) OA treatment increased the volume of TG in Huh7 cells. (D) Lipid droplets were significantly increased compared with untreated controls (Scale bar $=30 \mu \mathrm{M}$ ). (E) The volume of intracellular lipid droplets was also increased as determined through the quantification column. ${ }^{*} \mathrm{P}<0.05$ and ${ }^{* *} \mathrm{P}<0.01$. OA, oleic acid; BSA, bovine serum albumin; SREBP-1c, sterol regulatory element binding protein-1c; TG, triglyceride.

manufacturer's instructions. TG values are expressed as $\mu \mathrm{M}$ (at the cellular level).

Statistical analysis. Bar graphs depict means \pm standard deviation of at least three independent experiments. A Student's t-test was used to analyze differences between means for two independent samples, and $\mathrm{P}<0.05$ was considered to indicate a statistically significant difference.

\section{Results}

It has been reported that lncHR1 regulated SREBP-1c levels in Huh7 cells and transgenic mice fed a high fat diet (15). However, the detailed mechanism behind the regulation of SREBP-1c by lncHR1 has not been further studied. Therefore, we utilized a cells model of the TG accumulation induced by oleic acid (OA) and then studied the effects of IncHR1 on SREBP-1c.

LncHR1 inhibited SREBP-1c through regulation of AKT phosphorylation levels in the TG accumulation model. The cell model of OA-induced TG accumulation was used. The

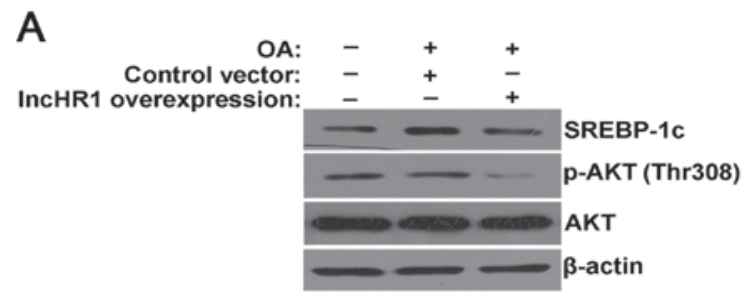

B

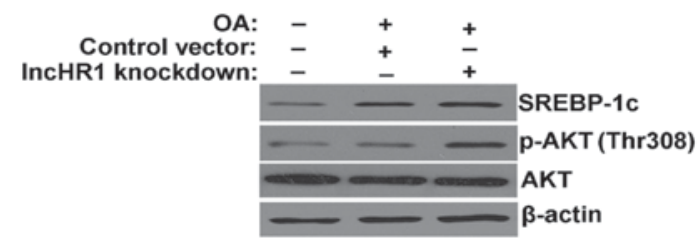

Figure 2. LncHR1 suppresses the AKT/ SREBP-1c pathway in the cellular model of Steatosis. (A) In the cellular model of the steatosis, overexpressed 1ncHR1 inhibited SREBP-1c protein levels and the phosphorylation of AKT (Thr308) level was suppressed. Total protein AKT and $\beta$-actin used as control. (B) Knockdown of lncHR1 elevated SREBP-1c protein levels and activated the phosphorylation of AKT (Thr308) in the steatosis cells. 1ncHR1, long non-coding RNA HCV regulated 1; SREBP-1c, sterol regulatory element binding protein-1c; OA, oleic acid. 
A

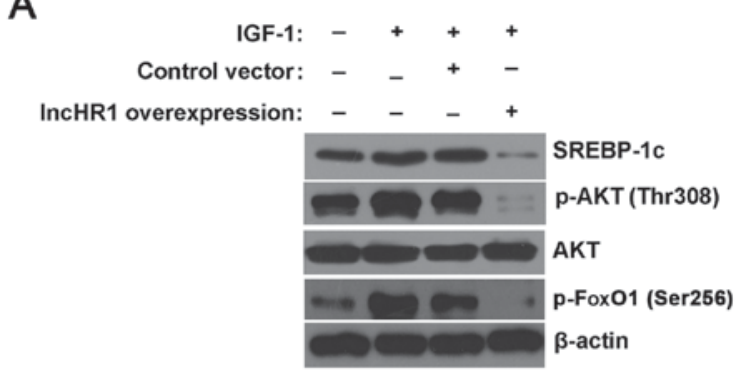

B

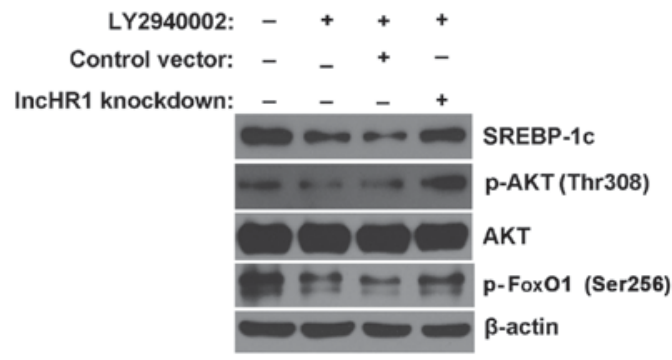

C

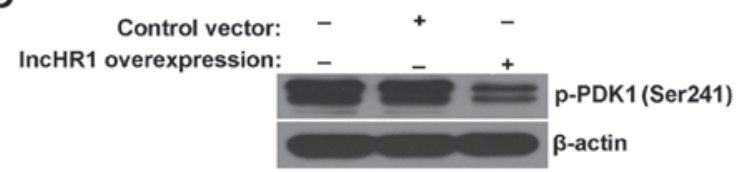

D

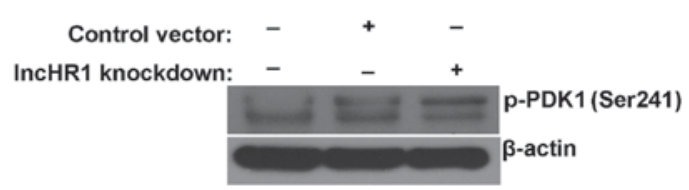

Figure 3. LncHR1 affects the p-PDK1/AKT/FoxO1/SREBP-1c pathway. (A) IGF-1, an activator of PI3K/AKT, simultaneously increased SREBP-1c levels and the phosphorylation level of AKT (Thr308) and FoxO1 (Ser256). This elevated phosphorylation was significantly reversed by overexpression of lncHR1. IGF-1 induction of the SREBP-1c protein by was also reversed following lncHR1 overexpression. Total AKT and $\beta$-actin were used as controls. (B) LY294002, an inhibitor of PI3K/AKT, suppressed AKT (Thr308) and FoxO1 (Ser256) phosphorylation compared to AKT and $\beta$-actin. LncHR1 knockdown rescued LY2940002 inhibition of AKT and FoxO1 phosphorylation, as well as SREBP-1c protein levels. (C) The phosphorylation level of PDK1 at Ser241 site was suppressed by lncHR1 overexpression. $\beta$-actin was used as a loading control. (D) LncHR1 knockdown activated the phosphorylation level of PDK1 (Ser241). $\beta$-actin was used as a loading control. lncHR1, long non-coding RNA HCV regulated 1; SREBP-1c, sterol regulatory element binding protein-1c; FoxO1, forkhead Box O1; PDK1, 3-phosphoinositide-dependent protein kinase 1.

results showed that, in Huh7 cells, OA treatment for $24 \mathrm{~h}$ obviously increased SREBP-1c protein (Fig. 1A) and mRNA level (Fig. 1B) compared to control cells. In addition, there was no obvious change in lncHR1mRNA levels (data not shown). In hepatic cells, higher SREBP-1c is conductive to the synthesis and accumulation of TG. Therefore, we analyzed the TG levels in the hepatic cell model. The results revealed an increase of TG in OA treated cells (Fig. 1C). In the liver, SREBP-1c is an activator of FAS, which enhances FA synthesis and accelerates TG accumulation (18). TG are normally stored in
A

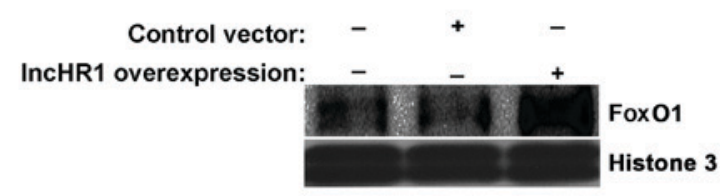

B

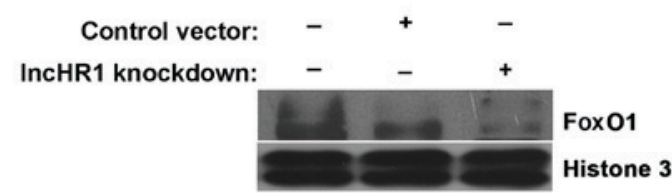

C

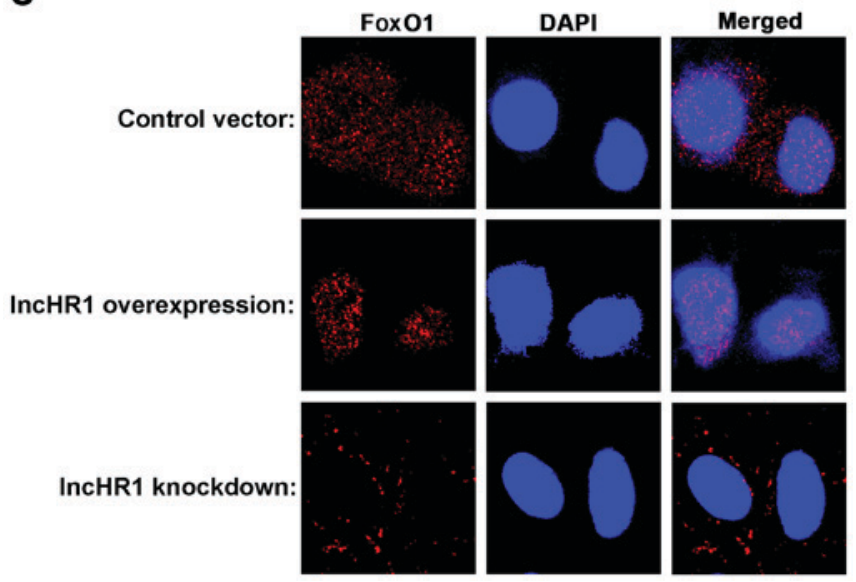

Figure 4. LncHR1 regulated the distribution of FoxO1 inside and outside of the nucleus. LncHR1 overexpression or knockdown plasmids were transfected into Huh7 cells. After 48 h post-transfection, the nuclear protein fraction was separated for western blotting. In the nucleus, FoxO1 was increased by 1 ncHR1 overexpression (A) and was reduced after lncHR1 knockdown. (B) Immunofluorescence staining showed that FoxO1 was increased in the nucleus by 1 chR 1 overexpression. (C) The accumulation of FoxO1 in the nucleus was reduced by knockdown lncHR1 (Scale bar=20 $\mu \mathrm{M})$. IncHR1, long non-coding RNA HCV regulated 1; FoxO1, forkhead Box O1.

the form of neutral lipid droplets in hepatocytes or secreted as TG-enriched lipoproteins into the bloodstream. Through the oil red O staining in morphology, we observed that OA treatment significantly increased lipid droplets compared with untreated controls (Fig. 1D). Consistently, the volume of intracellular lipid droplets was also increased (Fig. 1E), as determined via immunofluorescence assay. These data verified that the model of OA-induced hepatic cell TG accumulation. Therefore, this model can be used for subsequent studied into the regulation of SREBP-1c protein by lncHR1. The efficiency of overexpression or knockdown lncHR1 vectors was previously verified (15) and the two vectors were used in this experiment.

In the model of the TG accumulation, overexpression of lncHR1 inhibited SREBP-1c protein expression, suppressed the phosphorylation of AKT (Thr308) and total AKT protein levels. $\beta$-actin was used as a loading control (Fig. 2A). Alternatively, knockdown of lncHR1 elevated SREBP-1c protein levels and activated the phosphorylation of AKT (Thr308) (Fig. 2B). Therefore, our results suggested that lncHR1 may inhibit SREBP-1c through regulation of AKT phosphorylation in the model of the TG accumulation. As 
previously reported, FoxO1 is a member of the family of a forkhead box class-O transcription factor family and is a downstream target of serine/threonine protein kinase $\mathrm{B}$ (AKT). Research has confirmed that, in the promoter region of the srebp-1c gene, FoxO1 weakens the combinatorial capacity of LXR $\alpha /$ RXR binding to LXREs, subsequently inhibiting srebpl gene expression (10).

LncHRl affected the phosphorylation of PDK1/AKT/FoxO1 in Huh7 cells. To study how lncHR1 regulates the phosphorylation of AKT, an activator (IGF-1) and inhibitor (LY294002) of the PI3K/AKT pathway were used in this study. As shown in Fig. 3A, IGF-1 simultaneously increased the phosphorylation level of AKT (Thr308) and FoxO1 (Ser256), but this elevated phosphorylation was significantly reversed by overexpression of lncHR1. Meanwhile, induced expression of SREBP-1c by IGF-1 was also reversed after lncHR1 overexpression (Fig. 3A). As expected, the inhibitor LY294002 suppressed AKT (Thr308) and FoxO1 (Ser256) phosphorylation comparing to total AKT and $\beta$-actin. Nevertheless, lncHR 1 knockdown rescued LY2940002 inhibition of AKT and FoxO1 phosphorylation, as well as SREBP-1c protein (Fig. 3B). Thus, AKT (Thr308) and FoxO1 (Ser256) may be the key site of phosphorylation in regulation of the AKT/FoxO1/SREBP-1c axis by lncHR1. Therefore, the molecule upstream of AKT may be the targets of IncHR1. AKT is phosphorylated and activated by PI3K, which is composed of a heterodimer between a p110 catalytic subunit and a p85 regulatory subunit. The results showed that there was no change in the protein level of $\mathrm{p} 110 \beta$ or $\mathrm{p} 85$, regardless of whether lncHR1 was overexpressed or knocked down (data not shown). Therefore, the key site for $\operatorname{lncHR} 1$ regulation of the AKT/FoxO1/SREBP-1c axis is likely independent of PI3K.

The major biological function of phosphatase and tensin homolog (PTEN) relies on its phosphatase activity and PTEN exerts tumor suppressive functions by suppressing the PI3K pathway (19). In this study, the results showed that IncHR1 expression had no effect on PTEN levels, similar to the PI3K subunits (data not shown).

3-phosphoinositide-dependent protein kinase 1 (PDK1) is downstream of PI3K and activated PDK1 can stimulates the phosphorylation of Threonine 308 in the central catalytic domain (20). Western blotting data confirmed that PDK1 phosphorylation at Ser241 was suppressed by lncHR1 overexpression (Fig. 3C) and was activated by lncHR1 knockdown (Fig. 3D). However, the detailed mechanisms of how lncHR1 regulate the phosphorylation of PDK1 at site Ser241 requires further study.

LncHRl affected the distribution of FoxOl in Huh7 cells. A study by Kamei (9) also indicated that SREBP-1c gene expression was up-regulated by the combination of LXR $\alpha / \mathrm{RXR}$ bound to LXRE, and that FoxO1 antagonized this combination to inhibit srebp-1c gene expression.

Phosphorylation of FoxO1 (Ser256) (p-FoxO1) by AKT occurs in the cytoplasm. Non-phosphorylated FoxO1 located in the nucleus inhibits SREBP-1c expression. Therefore, Western blotting and immunofluorescence assays were used to measure the lncHR1-regulated translocation of FoxO1. First, we separated the nuclear protein fraction for each treatment and then quantified FoxO1 accumulation. The results indicated that a greater amount of FoxO1 accumulated in the nucleus when lncHR1 was overexpressed (Fig. 4A) and the opposite results were observed after lncHR1 knockdown (Fig. 4B). This effect on the subcellular translocation of FoxO1 was confirmed by immunofluorescent staining (Fig. 4C). Thus, lncHR1 suppressed phosphorylation of the AKT/FoxO1 level, increased the quantity of FoxO1 in the nucleus, and then downregulated SREBP-1c expression. LncHR1 may be a negative regulatory factor of SREBP-1c transcription.

Above all, these results consolidated our findings that lncHR1 suppressed SREBP-1c levels through decreased the phosphorylation level of PDK1/AKT/FoxO1 and increased expression of intranuclear FoxO1. According to these results, lncHR1 may inhibit the expression of SREBP-1c through modulation of the PDK1/AKT/FoxO1 pathway in Huh7 cells. Further studies will investigate how lncHR1 regulate the phosphorylation of PDK1.

\section{Discussion}

In this study, we found that lncHR1 inhibited SREBP-1c and TG accumulation when the phosphorylation level of the PDK1/AKT/FoxO1 pathway was reduced through increased lncHR1. Based on the results of previous experiments (15), we initially concluded that lncHR 1 participated in the balance metabolism of lipid through regulating the phosphorylation level of the PDK1/AKT/FoxO1 signaling pathway, thus affecting SREBP-1c and TG accumulation.

SREBPs are key transcriptional factors that control lipogenesis and lipid uptake (21). SREBP-1c activates the transcription of multiple genes encoding for enzymes involved in the synthesis of cholesterol, TG, and phospholipids, which are related to FA synthesis (22). Some small molecular that regulate SREBP levels may affect lipid metabolism. For example, miR-33a is a highly conserved intronic miRNA, the level of which is increased in normal tissues by increased transcription of SREBF2, resulting in coordinate regulation of cholesterol and other lipid levels by SREBF2 and miR-33a (23). MALAT1 induced hepatic lipid accumulation and insulin resistance by increasing SREBP-1c and target genes expression (16). Our previous study (15) had confirmed that lncHR1 indeed simultaneously regulated the SREBP-1c levels and TG and lipid droplets in vivo.

The long non-coding RNA (lncRNA) urothelial carcinoma-associated 1 (UCA1) showed significantly higher expression in advanced gastric cancer tissues, and the study results indicated that UCA1 regulates PI3K-AKT-mTOR signaling proteins in vitro and in vivo (24).

In particular, SREBP-1c is the chief factor regulating the transcription of genes involved in fat synthesis, and can be up-regulated by $\operatorname{LXR} \alpha$ (21). LXR $\alpha$ heterodimerized with the nuclear receptors retinoid X receptors (RXRs) and binds to the LXR element (LXRE) in the upstream promoter of srebpl genes, thus controlling the transcription of SREBP-1c and further regulating the expression of the downstream genes involved in de novo fatty acid metabolism, TG synthesis and cholesterol homeostasis (25). As reported previously, FoxO1 antagonized the binding of LXR $\alpha /$ RXR to LXRE and lowered srebp-1c gene expression to some extent. FoxO1, a member of the FoxO family of transcription factors, is the direct downstream substrates for AKT. When phosphorylated by AKT, FoxO1 translocate from 
the nucleus to the cytoplasm. In the current study, our results indicated that either overexpression or knockdown lncHR1 may affect the distribution of FoxO1 inside and outside of the nucleus (Fig. 4).

Activation of the PI3K/AKT signaling pathway results in AKT-dependent phosphorylation of FoxO1, reducing FoxO1 nuclear translocation and inhibiting its transcriptional function. PI3K/AKT signaling upregulates glucose uptake and glycolysis and controls the metabolic flux from glucose and glutamine to de novo lipid synthesis (26).

SREBP-1c may be activated by the PI3K/AKT oncogenic signaling pathway either PI3K/AKT/GSK3- $\beta$ /SREBP-1c (27) or PI3K/AKT/mTORC1 signaling (28). However, our previous studies did not confirm that lncHR1 was involved in the classical pathway. Activated PDK1 stimulates AKT activity by direct binding and phosphorylation of Threonine 308 in the central catalytic domain (20). In our study, the results (Fig. 3) indicated that lncRH1 participated in the phosphorylation of PDK1, subsequently regulating the AKT/FoxO1/SREBP-1c pathway. Multiple studies have revealed that PI3K/AKT signaling promotes fatty acid synthesis through upregulation of SREBP-1c (29). A study by Wang indicated that IncRNA AB073614 may be useful as a novel prognostic or treatment agent for colorectal cancer due to its effects on the PI3K/AKT signaling pathway (30).

It is well established that the major biological function of PTEN relies on its phosphatase activity. PTEN dephosphorylates PIP3 to PIP2, thereby inhibiting the PI3K signaling pathway (19). However, our results did not support the theory that lncHR1 influences PTEN or involvement in this phosphorylation process (data not shown).

There are several limitations in this study. i) The detailed molecular mechanism for the effect of lncHR1 on the phosphorylation of PDK1 has not been elucidated. ii) The phosphorylation of AKT is regulated by mTOR, but whether lncHR1 is involved in the mTOR pathway was not verified. Therefore, these areas will be the next focus of our research.

Taken together, these findings indicated that lncHR1 may induce a decrease in the phosphorylation of the PDK1/AKT/FoxO1 pathway and suppressed SREBP-1c protein levels. However, the precise role of 1 chR 1 in the phosphorylation of PDK1 remains to be elucidated, and further investigations is needed.

\section{Acknowledgements}

The authors would like to thank Dr Wei Yang of the MOH Key Laboratory of Systems Biology of Pathogens, Institute of Pathogen Biology, Chinese Academy of Medical Sciences and Peking Union Medical College for providing technical and material support.

\section{Funding}

This work was supported by the Collective grants from the Programs for Science and Technology Development of Henan (grant nos. 172102310499 and 182102310240), Open Program of Henan Key Laboratory of Biological Psychiatry (grant no. ZDSYS2016007) and Dr. scientific research start-up fund (grant no. XYBSKYZZ201605).

\section{Availability of data and materials}

The analyzed data sets analyzed during the study are available from the corresponding author on reasonable request.

\section{Authors' contributions}

DL and ZY are responsible for the study concept and design. LG and BD performed part of the cell biology experiment. ML, TY and FY performed the molecular biology experiment. DL and $\mathrm{ZY}$ were involved in the data analysis and manuscript drafting.

\section{Ethics approval and consent to participate}

Not applicable.

\section{Patient consent for publication}

Not applicable.

\section{Competing interests}

The authors declare that they have no competing interests.

\section{References}

1. Brown MS and Goldstein JL: The SREBP pathway: Regulation of cholesterol metabolism by proteolysis of a membrane-bound transcription factor. Cell 89: 331-340, 1997.

2. Si Y, Liu S, Liu X, Jacobs JL, Cheng M, Niu Y, Jin Q, Wang T and Yang W: A human claudin-1-derived peptide inhibits hepatitis C virus entry. Hepatology 56: 507-515, 2012.

3. Horton JD, Bashmakov Y, Shimomura I and Shimano H: Regulation of sterol regulatory element binding proteins in livers of fasted and refed mice. Proc Natl Acad Sci USA 95: 5987-5992, 1998.

4. Repa JJ, Liang G, Ou J, Bashmakov Y, Lobaccaro JM, Shimomura I, Shan B, Brown MS, Goldstein JL and Mangelsdorf DJ: Regulation of mouse sterol regulatory element-binding protein-1c gene (SREBP-1c) by oxysterol receptors, LXRalpha and LXRbeta. Genes Dev 14: 2819-2830, 2000.

5. Horton JD, Goldstein JL and Brown MS: SREBPs: Activators of the complete program of cholesterol and fatty acid synthesis in the liver. J Clin Invest 109: 1125-1131, 2002.

6. Ferré P and Foufelle F: SREBP-1c transcription factor and lipid homeostasis: Clinical perspective. Horm Res 68: 72-82, 2007.

7. Chen G, Liang G, Ou J, Goldstein JL and Brown MS: Central role for liver $\mathrm{X}$ receptor in insulin-mediated activation of Srebp-1c transcription and stimulation of fatty acid synthesis in liver. Proc Natl Acad Sci USA 101: 11245-11250, 2004.

8. Deng X, Yellaturu C, Cagen L, Wilcox HG, Park EA, Raghow R and Elam MB: Expression of the rat sterol regulatory element-binding protein-1c gene in response to insulin is mediated by increased transactivating capacity of specificity protein 1 (Sp1). J Biol Chem 282: 17517-17529, 2007.

9. Kamei Y, Miura S, Suganami T, Akaike F, Kanai S, Sugita S, Katsumata A, Aburatani H, Unterman TG, Ezaki O and Ogawa Y: Regulation of SREBP1c gene expression in skeletal muscle: Role of retinoid $\mathrm{X}$ receptor/liver $\mathrm{X}$ receptor and forkhead-O1 transcription factor. Endocrinology 149: 2293-2305, 2008.

10. Deng X, Zhang W, O-Sullivan I, Williams JB, Dong Q, Park EA, Raghow R, Unterman TG and Elam MB: FoxO1 inhibits sterol regulatory element-binding protein-1c (SREBP-1c) gene expression via transcription factors Sp1 and SREBP-1c. J Biol Chem 287: 20132-20143, 2012.

11. Johnsson P, Lipovich L, Grandér D and Morris KV: Evolutionary conservation of long non-coding RNAs; sequence, structure, function. Biochim Biophys Acta 1840: 1063-1071, 2014.

12. Gibb EA, Brown CJ and Lam WL: The functional role of long non-coding RNA in human carcinomas. Mol Cancer 10: 38, 2011.

13. Maass PG, Luft FC and Bahring S: Long non-coding RNA in health and disease. J Mol Med (Berl) 92: 337-346, 2014. 
14. Panzitt K, Tschernatsch MM, Guelly C, Moustafa T, Stradner M, Strohmaier HM, Buck CR, Denk H, Schroeder R, Trauner M and Zatloukal K: Characterization of HULC, a novel gene with striking up-regulation in hepatocellular carcinoma, as noncoding RNA. Gastroenterology 132: 330-342, 2007.

15. Li D, Cheng M, Niu Y, Chi X, Liu X, Fan J, Fan H, Chang Y and Yang W: Identification of a novel human long non-coding RNA that regulates hepatic lipid metabolism by inhibiting SREBP-1c. Int J Biol Sci 13: 349-357, 2017.

16. Yan C, Chen J and Chen N: Long noncoding RNA MALAT1 promotes hepatic steatosis and insulin resistance by increasing nuclear SREBP-1c protein stability. Sci Rep 6: 22640, 2016.

17. Cheng M, Si Y, Yang Y, Liu X, Gong Q, Zhao J, Niu Y, Li X, Jin Q and Yang W: Recombinant human interleukin 28B: Anti-HCV potency, receptor usage and restricted cell-type responsiveness. J Antimicrob Chemother 67: 1080-1087, 2012.

18. Latasa MJ, Moon YS, Kim KH and Sul HS: Nutritional regulation of the fatty acid synthase promoter in vivo: Sterol regulatory element binding protein functions through an upstream region containing a sterol regulatory element. Proc Natl Acad Sci USA 97: 10619-10624, 2000.

19. Worby CA and Dixon JE: PTEN. Annu Rev Biochem 83: 641-669, 2014

20. Franke TF, Kaplan DR, Cantley LC and Toker A: Direct regulation of the Akt proto-oncogene product by phosphatidylinositol-3,4-bisphosphate. Science 275: 665-668, 1997.

21. Jianhua L, Xueqin M and Jifen H: Expression and clinical significance of LXR $\alpha$ and SREBP-1c in placentas of preeclampsia. Open Med (Wars) 11: 292-296, 2016.

22. Horton JD, Shimomura I, Ikemoto S, Bashmakov Y and Hammer RE: Overexpression of sterol regulatory element-binding protein-1a in mouse adipose tissue produces adipocyte hypertrophy, increased fatty acid secretion, and fatty liver. J Biol Chem 278: 36652-36660, 2003.

23. Najafi-Shoushtari SH, Kristo F, Li Y, Shioda T, Cohen DE, Gerszten RE and Näär AM: MicroRNA-33 and the SREBP host genes cooperate to control cholesterol homeostasis. Science 328: $1566-1569,2010$
24. Li C, Liang G, Yang S, Sui J, Yao W, Shen X, Zhang Y, Peng H, Hong W, Xu S, et al: Dysregulated IncRNA-UCA1 contributes to the progression of gastric cancer through regulation of the PI3K-Akt-mTOR signaling pathway. Oncotarget 8: 93476-93491, 2017.

25. Plösch T, Gellhaus A, van Straten EM, Wolf N, Huijkman NC, Schmidt M, Dunk CE, Kuipers F and Winterhager E: The liver $\mathrm{X}$ receptor (LXR) and its target gene ABCA1 are regulated upon low oxygen in human trophoblast cells: A reason for alterations in preeclampsia? Placenta 31: 910-918, 2010.

26. Metallo CM, Gameiro PA, Bell EL, Mattaini KR, Yang J, Hiller K, Jewell CM, Johnson ZR, Irvine DJ, Guarente L, et al: Reductive glutamine metabolism by IDH1 mediates lipogenesis under hypoxia. Nature 481: 380-384, 2011.

27. Yecies JL, Zhang HH, Menon S, Liu S, Yecies D, Lipovsky AI, Gorgun C, Kwiatkowski DJ, Hotamisligil GS, Lee CH and Manning BD: Akt stimulates hepatic SREBP1c and lipogenesis through parallel mTORC1-dependent and independent pathways. Cell Metab 14: 21-32, 2011.

28. Peterson TR, Sengupta SS, Harris TE, Carmack AE, Kang SA, Balderas E, Guertin DA, Madden KL, Carpenter AE, Finck BN and Sabatini DM: mTOR complex 1 regulates lipin 1 localization to control the SREBP pathway. Cell 146: 408-420, 2011.

29. Porstmann T, Santos CR, Griffiths B, Cully M, Wu M, Leevers S, Griffiths JR, Chung YL and Schulze A: SREBP activity is regulated by mTORC1 and contributes to Akt-dependent cell growth. Cell Metab 8: 224-236, 2008

30. Wang Y, Kuang H, Xue J, Liao L, Yin F and Zhou X: LncRNA AB073614 regulates proliferation and metastasis of colorectal cancer cells via the PI3K/AKT signaling pathway. Biomed Pharmacother 93: 1230-1237, 2017. 